\title{
Prospects for Scrutiny of Pulsars with Polish Part of LOFAR
}

\author{
Leszek BŁASZKIEWICZ ${ }^{1,3}$, Wojciech LEWANDOWSKI ${ }^{2}$, \\ Andrzej KRANKOWSKI ${ }^{1}$, Jarosław KIJAK ${ }^{2}$, Olga KORALEWSKA ${ }^{2}$, \\ and Bartosz P. DĄBROWSKI ${ }^{1}$ \\ ${ }^{1}$ Space Radio-Diagnostics Research Centre, \\ University of Warmia and Mazury, Olsztyn, Poland; \\ e-mail: kand@uwm.edu.pl \\ ${ }^{2}$ Institute of Astronomy, University of Zielona Gora, \\ Zielona Góra, Poland; \\ e-mail: boe@astro.ia.uz.zgora.pl \\ ${ }^{3}$ Faculty of Mathematics and Computer Sciences, \\ University of Warmia and Mazury, Olsztyn, Poland; \\ e-mail: leszekb@matman.uwm.edu.pl (corresponding author)
}

\begin{abstract}
LOFAR (LOw Frequency ARray) is a new generation digitally controlled radio telescope consisting of phased array antenna stations with sensitivity, bandwidth, range of frequency, and digital processing power that makes it an excellent tool for observations of pulsars. This interferometric instrument is able to work in a single-station mode as well as in group-of-selected-stations mode. This article discusses the great opportunity for conducting unique and independent research of pulsar sources with the three LOFAR stations located in Poland and maintained by the POLFAR consortium.
\end{abstract}

Key words: radio astronomy, LOFAR, pulsar, neutron star.

Ownership: Institute of Geophysics, Polish Academy of Sciences;

(C) 2015 Błaszkiewicz et al. This is an open access article distributed under the Creative Commons Attribution-NonCommercial-NoDerivs license,

http://creativecommons.org/licenses/by-nc-nd/3.0/. 


\section{INTRODUCTION}

During the first two decades of radio astronomical research, after the discovery of radio emission from the Milky Way (Jansky 1933), research was concentrated on observations at frequencies below a few hundred $\mathrm{MHz}$. However, since the first detection of the $21 \mathrm{~cm}$ neutral hydrogen line at $1.420 \mathrm{GHz}$ (Ewen and Purcell 1951) the observations at higher frequencies became the dominating source of new information. It was only in the 1990s that radio astronomy came back to its roots and returned to exploring the long radio waves. The advances in computer technology allowed for detailed studies of the Universe using interferometric methods. This was also helped by a better knowledge of the Earth's ionosphere, a layer of the Earth's atmosphere that interacts with the low frequency cosmic radio waves causing distortions in its wavefront - and these have to be disentangled from the observational data to retrieve useful information. One of the main instruments that attempts to do this is the Low Frequency Array (LOFAR). LOFAR is an instrument that uses phased array technologies that are able to operate at low radio frequencies. It is worth mentioning that one of the key science projects for LOFAR telescope - from early was the search for and observations of transient radio sources, with a particular stress on pulsar studies (Stappers et al. 2007).

First detection of a pulsar signal was made in the mid-60s at the observing frequency of $81 \mathrm{MHz}$ by Hewish and collaborators (history of pulsar research, methods of observation, and their physics are described in detail by Lorimer and Kramer 2005), and it was soon discovered that the pulsating radio signal that was detected is generated by a rapidly rotating neutron star with extremely high magnetic field. The electromagnetic spectrum of emissions from a neutron star gives the opportunity to observe pulses also at optical, $x$ - and $\gamma$-rays frequencies. However, radio frequencies are the area of the spectrum where pulsar emissions are easiest to detect and can be analyzed in great detail.

Nearly half a century of pulsar research with radio astronomical methods allowed us to develop a reasonably acceptable model explaining the properties of pulsar radiation - the crucial information about these is presented in Section 3. Nevertheless, there are many questions and details of the physical properties of pulsars which still remain unanswered. In particular, the properties of pulsar low-frequency emissions were - until now - only rarely studied with the high time and spatial resolution that the use of modern technology allows.

Nowadays, after early LOFAR observations of pulsars (Hessels et al. 2010) and latter detailed study of selected pulsars (e.g., Bilous et al. 2014) we can be certain that LOFAR is currently one of the best instruments that 
can be used to study the pulsar's radio signals in great detail, at a level that was previously unattainable.

The main purpose of this article is to present a new instrument and its unique properties that will be very advantageous in progressive studies of pulsars as well as in other fields of radio-astrophysics. It is the first of a series of two papers that are presenting research plans associated with the Polish part of the LOFAR telescope. Pulsars will be one of the leading research topics of POLFAR, and this article introduces the Polish pulsar team, which combines researchers from the Space Radio-Diagnostics Research Centre from UWM Olsztyn and from the Institute of Astronomy of the University of Zielona Góra.

\section{THE LOFAR TELESCOPE}

The LOFAR system design, configuration and the signal processing methods were described in detail by de Vos et al. (2009) and van Haarlem et al. (2013). The main idea of a single station phased array, as well as the operation principle of whole LOFAR interferometer are presented in Fig. 1. Below we present only the most important characteristics of the instrument and its functions.

(a)
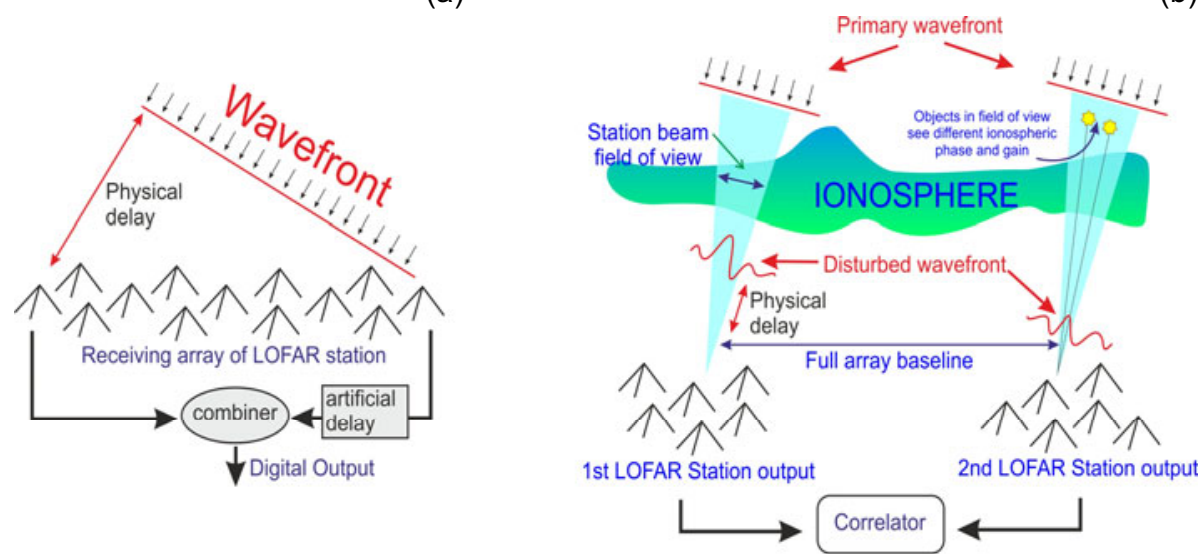

Fig. 1: (a) The LOFAR single station as the phased array antenna; digital control allows to direct the beam to any part of the sky; (b) signal from many different stations goes to a correlator. The influence of the ionosphere on the signal wavefront distortion must necessarily be taken into account during correlation. The right part of the image was inspired by van der Tol and van der Veen (2007).

\subsection{LOFAR design and operation}

The LOFAR interferometer consists of a large number (>100 000) of omnidirectional dipole antennas, that will be arranged to form over 52 individual 
stations across Europe in the close future. The LOFAR receivers' bandwidth spreads from 10 to $240 \mathrm{MHz}$, but actually excludes the frequency range from 88 up to $108 \mathrm{MHz}$ because of the expected Earth-bound interferences. Each individual station consists of two antenna fields: (1) the Low Band Antennas (LBAs) which are sensitive to the wavelengths from 4 to $10 \mathrm{~m}(10-90 \mathrm{MHz})$ (a single station LBA field contains 48 or 96 antennas), antennas of the LBA field in full configuration occupy an area with a radius of about $40 \mathrm{~m}$; (2) the High Band Antennas (HBAs) are collected in tiles, and operate in the wavelength range between 1 and $3 \mathrm{~m}(110-250 \mathrm{MHz})$ (again, 48 or 96 tiles per station). In the full 96 tiles configuration the diameter of the HBA field is $62 \mathrm{~m}$. The configuration of a typical international LOFAR station is shown in Fig. 2.

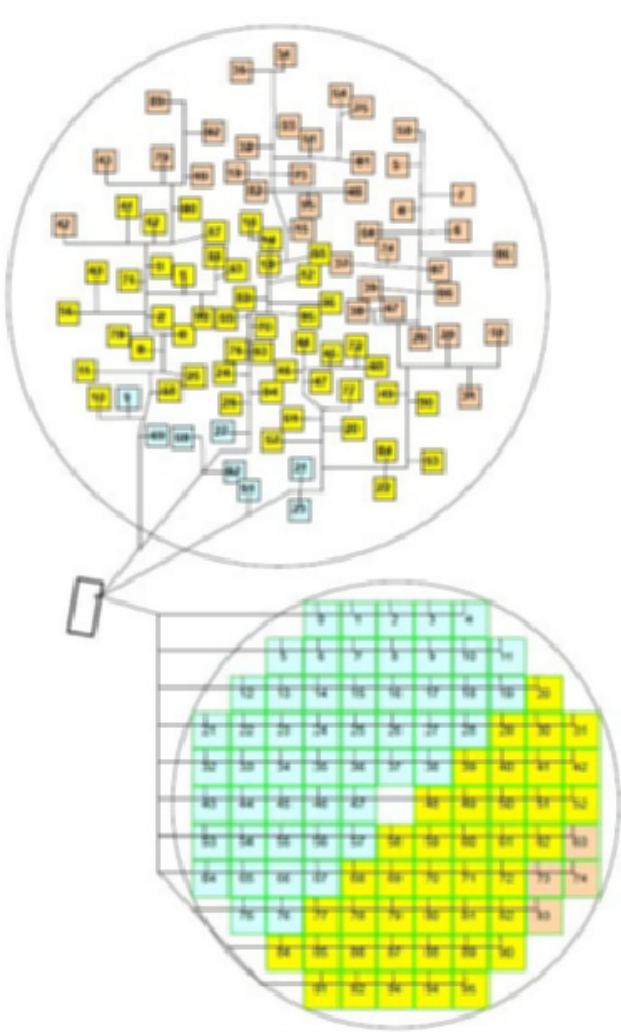

(a)

(b)

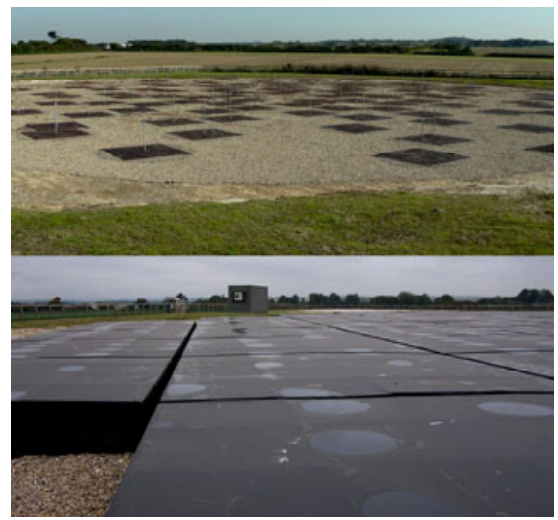

Fig. 2: (a) The configuration of an international LOFAR station; the larger upper circle represents the LBA antennas and the lower one depicts HBA tiles (figure taken from van Haarlem et al. 2013); (b) the ground view of the international station UK608 (Chilbolton): upper photo - the LBA antennas, bottom photo - the HBA tiles. Photos: L. Błaszkiewicz. 
At the single station level the signals received by individual dipoles are digitally combined to form a phased array, by introducing time delays to the signals in a process which is known as beam-forming. Doing so mimics a single telescope dish, and such a virtual telescope can be steered electronically by adjusting the delays for individual receiver dipoles, which allows to "point" the telescope at any object on the sky and follow it in its daily motion.

Each individual station is connected via an optic fiber network link to a datacenter. The data transfer capability of $10 \mathrm{Gbits} / \mathrm{s}$ allows for a continuous data transmission in 244 sub-bands. Each of these sub-bands allows for the creation of an independent beam, which then can be pointed at the required position in the sky. The idea of multibeam observations - which is very useful for pulsar investigations as it allows for observations of a few objects simultaneously - is shown in Fig. 3.

To this day the LOFAR network consists of 49 stations - most of them in the Netherlands, with 6 stations in Germany and one station each in UK, France, and Sweden - which are finished and in operation, and the 3 Polish stations, which are under construction. The Polish part of LOFAR, called POLFAR, has been described by Krankowski et al. (2014).

The majority of the stations are located in the Netherlands, including 24 stations located within a radius of about $2 \mathrm{~km}$ called "core", and a round island of $320 \mathrm{~m}$ diameter referred as "Superterp" in the middle of the core region.

The remaining 16 Dutch stations referred to as "remote" are located beyond the core in a distance of up to $90 \mathrm{~km}$ and placed more or less (depending on land availability) in a logarithmic spiral distribution.

(a)

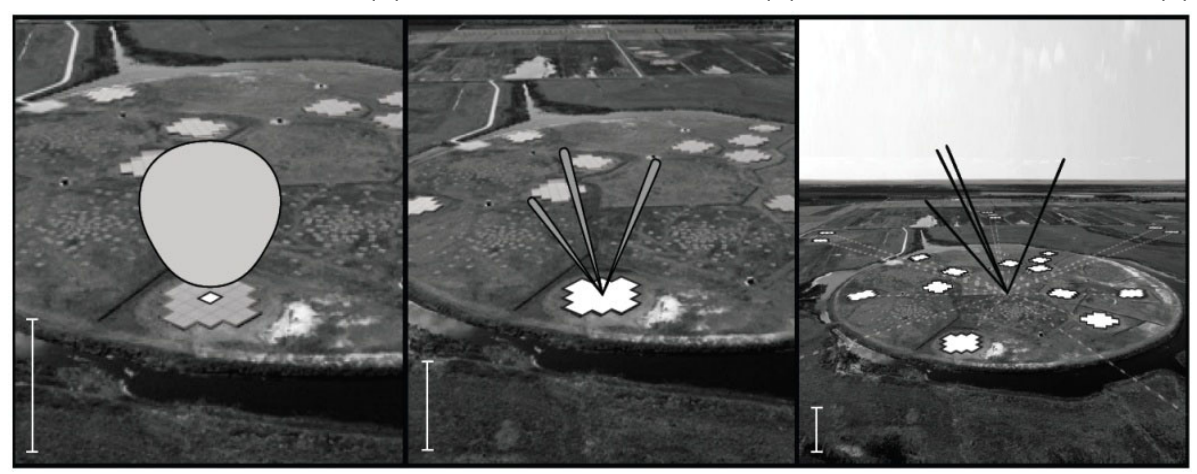

Fig. 3. The multibeam forming idea shown for the HBA part of the LOFAR core and Superterp area (the circular island): (a) single element beam, (b) single station, and (c) tied array for all 24 core stations. Figure taken from Stappers et al. (2011). 
A full list of stations (excluding the newest one at Norderstedt) with accurate coordinates is available in Table A1 in van Haarlem et al. (2013).

The individual station outputs are split into narrow frequency bins, correlated, averaged over short intervals, and stored for offline processing. The whole instrument with a longest baseline of about $1000 \mathrm{~km}$ allows for an angular resolution close to $0.21 \mathrm{arcsec}$ at $240 \mathrm{MHz}$ and about 3.3 arcsec for the longest waves (at $15 \mathrm{MHz}$ ). The sensitivity of LOFAR core with 8 hours of integration and an effective bandwidth of $3.66 \mathrm{MHz}$ at 2 polarizations is about $9 \mathrm{mJy}$ at $30 \mathrm{MHz}$ and $0.6 \mathrm{mJy}$ at $200 \mathrm{MHz}$ (see van Haarlem et al. 2013). In the case of an independent use of the three Polish stations (as a separated interferometer) the expected angular resolution could be about $1 \operatorname{arcsec}$ (resolution of LOFAR is given by $\alpha \lambda / L$, where $L$ denotes longest baseline, $\alpha=0.8$ and $\lambda$ is wavelength in meters; van Haarlem et al. 2013).

\subsection{Signal processing}

The very high spectral and time resolutions required in the observations of transient sources (such as pulsars) and the large analog-to-digital converters range will generate up to $13 \mathrm{Tbits} / \mathrm{s}$ of raw data by the whole LOFAR array when using a typical $200 \mathrm{MHz}$ sampling rate. For a single station processing this amount reduces to about $150 \mathrm{Gbits} / \mathrm{s}$. The storage space requirements are very demanding as well: one hour of interferometric imaging observations yields about 35 Tbyte of correlated visibilities. This amount of raw data requires a high computing power real time analysis and correlation and a huge disc space storage.

Until 2014 the IBM Blue Gene/P supercomputer located in the computing center of University of Groningen offered about 28 Tflops of processing power. Currently the correlation for interferometric imaging, tied-array beam forming for high time resolution observations and other computationally demanding modes of observation are handled by the COBALT system. It is a cluster of 32 extremely efficient NVIDIA Tesla GPUs. The description of the new correlation system can be found in a paper by Broekema (2013).

\subsection{POLFAR as a part of LOFAR}

The collaboration of the proposers from Poland is organized in the Polish Consortium LOFAR (POLFAR) and was established in 2007. The agreement was signed by the representatives of the Jagiellonian University (JU), the Space Research Center of Polish Academy of Sciences (SRC PAS), the University of Warmia and Mazury (UWM), the Nicolaus Copernicus University, the University of Zielona Góra, the Nicolaus Copernicus Astronomy Centre of Polish Academy of Sciences, the University of Szczecin, the Uni- 
versity of Environmental and Life Sciences, and the PIONIER company Polish Optical Internet (Krankowski et al. 2014).

Three institutions from the list (JU, SRC PAS, and UWM) are the constructors and owners of Polish LOFAR stations. By the end of 2015 all three LOFAR stations in Poland: in Bałdy near Olsztyn, Borówiec near Poznań, and Łazy near Kraków will be operational (see Table 1). The data from all our stations will be sent online to Poznań via fast $(\sim 10 \mathrm{~Gb} / \mathrm{s})$ PIONIER network and then to the correlator in the Netherlands. Additionally, the three stations of POLFAR form the smallest number that allows for the operation as an independent interferometer when they are not used as a part of the full LOFAR system.

Table 1

The summary for three Polish stations of LOFAR telescope

\begin{tabular}{|l|c|c|c|}
\hline \multicolumn{1}{|c|}{ Station name } & Bałdy/Olsztyn & Borówiec/Poznań & Lazy/Kraków \\
\hline LOFAR code & PL612 & PL610 & PL611 \\
Longitude & $20^{\circ} 35^{\prime} 26^{\prime \prime} \mathrm{E}$ & $17^{\circ} 04^{\prime} 28^{\prime \prime} \mathrm{E}$ & $20^{\circ} 29^{\prime} 23^{\prime \prime} \mathrm{E}$ \\
Latitude & $53^{\circ} 35^{\prime} 45^{\prime \prime} \mathrm{N}$ & $52^{\circ} 16^{\prime} 37^{\prime \prime} \mathrm{N}$ & $49^{\circ} 57^{\prime} 53^{\prime \prime} \mathrm{N}$ \\
Number of LBA elements & 96 & 96 & 96 \\
Number of HBA elements & 96 & 96 & 48 \\
Core distance [km] & 920 & 710 & 930 \\
\hline
\end{tabular}

\subsection{The specific back-end system for pulsar data analysis in POLFAR stations}

One of the main observational tasks related to LOFAR stations which has just formed in Poland (apart from the tasks related to the operation of the entire LOFAR network) will be the detection and analysis of pulsars signals in a single station mode. This kind of observations of transient events requires specialized hardware equipment as well as specific, dedicated software. The details of the online processing for pulsar observations with a LOFAR core are described in Stappers et al. (2011).

All the stations of the International LOFAR Telescope (ILT) are linked to a correlation center in the Netherlands through a broadband $10 \mathrm{~Gb}$ network. However, when not operating as a part of ILT, the data from an individual station may also be transferred to any local analyzing system.

Currently there are two transient-dedicated hardware/software solutions that can be implemented at each station. The first kind of backend used for analysis of LOFAR data from a single station in pulsar studies is ARTEMIS (Advanced Radio Transient Event Monitor and Identification System). It is the combined hardware/software tool using NVIDIA GPUs as an LOFAR 
data stream processor able to work at $3.2 \mathrm{Gbits} / \mathrm{s}$ data flow speed with $5 \mu \mathrm{s}$ sampling at two polarizations (Serylak et al. 2012). This method, for example, is used in the UK608 Chilbolton station and the FR606 Nancay site.

The second way, chosen for the POLFAR stations in Bałdy and Borówiec was previously adapted for ILT stations associated in the GLOW (German LOng Wavelength) Consortium. The Polish system will be based on computers with complex and potent architecture (two Xeon E5-2660v3, $128 \mathrm{~GB}$ of RAM and HDD with $18 \mathrm{~TB}$ of space). The data recording system for pulsar single LOFAR station use, called LuMP, was developed in Effelsberg. The DSPSR format of data as well as many other useful features was developed recently and is constantly being improved (see, e.g., Anderson 2012). The LuMP software will give the POLFAR community an opportunity for not only pulsar research (timing, polarization) but also allow for work in the spectrometer mode and data correlation between POLFAR stations. This opens the possibility of independent interferometric observations using LOFAR station in Poland alone.

The standard methods of data analysis will be based on the PSRCHIVE package. This is an open-source scientific data analysis software library with the application suite prepared for pulsar astronomy (van Straten et al. 2012).

\section{THE PRESENT STATE OF PULSAR INVESTIGATIONS}

Almost 50 years have passed since the discovery of pulsars (Hewish et al. 1968; Nobel Prize in Physics 1974), but still we have a lot to learn about these objects. Their basic properties and models for both the structure and the emission mechanisms are constantly under dispute. Also, since pulsars can be considered - to a large degree - to be very stable clocks (due to extremely stable rotation), they are being used as probes of the extreme conditions they sometimes find themselves in. Among these is the extremely strong gravity in tight binary systems, which actually allows for tests of various theories of gravity. This provided us with the means to confirm the existence of the emission of gravitational waves, which was predicted by Einstein's General Relativity, by the means of pulsar timing observations of PSR B1913+16 (see Hulse and Taylor 1975; Nobel Prize in Physics 1993). Also, the pulsed signals from these neutron stars can be excellent probes of the interstellar medium (ISM), both in case of the immediate environments of neutron stars (such as supernova remnant nebulae, or pulsar wind nebulae), as well as the general ISM in the Milky Way galaxy.

\subsection{Basic properties of pulsars}

Pulsars are born as the final products of evolution of massive stars (of at least 8 solar masses). Towards the end of their evolution, such stars start ac- 
cumulate iron nuclei in their cores. Since iron nuclei are the nuclei with the highest binding energy, they will not enter into reactions of nucleosynthesis. After enough iron is accumulated in the core of such a star, the thermonuclear reactions cease and the star - which was until this moment in a state of hydrostatic equilibrium between the gravity and thermal (and radiative) pressure - starts to collapse. The increase in density, pressure, and temperature of the collapsing core causes the star to explode as a supernova, expelling the outer layers into space. In the collapsing core the iron nuclei are disintegrated, and matter in a form of protons, neutrons, and electrons undergoes a process of neutronization (protons and electrons merge forming neutrons, with the excess energy being carried-out by neutrinos). If the mass of the stellar core was not too large, the collapse can be stopped by the pressure of newly formed neutrons.

This roughly sketched scenario explains the basic properties of neutron stars. They are small (radius of about $10 \mathrm{~km}$ ) balls of a very dense matter with a mass of about 1.5 solar masses the average density of a neutron star is comparable to the nuclear density. Newly born neutron stars spin rapidly (the initial spin periods are of the order of 10 milliseconds), which is a consequence of the conservation of angular momentum of the collapsing stellar core. They are also highly magnetized (up to $10^{12}$ gauss, $10^{14}$ in case of magnetars) which is also - at least partially - due to the collapse and the resulting compression of the initial magnetic field.

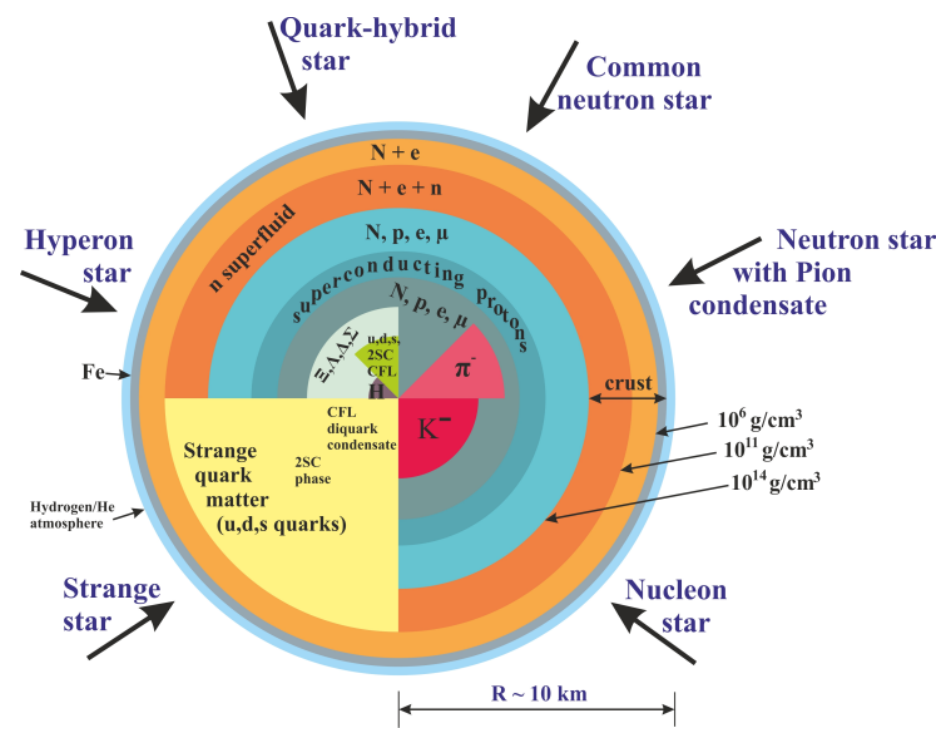

Fig. 4. The present status of theoretical study on neutron star interior. Figure based on Weber et al. (2009) elaboration. 
The structure of the neutron star interiors is still a field of intensive research and modelling. The extreme density conditions inside a neutron star are a challenge for modern physics. There are several models of the internal structure, involving various compositions and as a result requiring various equations of state (see, e.g., Shapiro and Teukolsky 1983, Pines and Alpar 1985). Figure 4 shows the most common models of the neutron star interiors.

\subsection{Pulsars' radio emission properties}

One of the basic properties of pulsars that have been observed since the very beginning is the fact that their rotation slows down. This is a common and expected feature since pulsars have to comply with the principles of the conservation of both energy and angular momentum, and still we see the outgoing radiation. This is why most of the observed neutron stars can be described as rotation-powered pulsars.

The pulsar magnetosphere, which is (roughly) dipolar in structure, is presumably rigidly rotating with the pulsar itself. Since, however, the pulsars are very fast rotators, at a certain distance from the rotational axis the velocity of the co-rotating magnetosphere would reach the speed of light (the distance at which it happens defines the so-called "light cylinder"). Only the magnetic fields lines that are in their entirety located within that distance can be closed, the remaining field lines will be "open" (Goldreich and Julian 1969). The particles in the magnetosphere can be accelerated only along the open field lines, and the region on the surface of neutron star where those open field lines originate is called a "polar cap" (see Fig. 5).

The mechanism of the pulsar's radio emission is still under investigation. Most of the particle acceleration (mainly electron-positron pairs) apparently happens just above the polar cap. One of the more successful models for this process, the Partially Screened Gap model, was presented by Gil et al. (2003) (see also Melikidze et al. 2014, Szary et al. 2015). These accelerated particles quickly loose most of their energy by emitting high-energy photons, which in turn - by the means of various cascade processes - can create additional electron-positron pairs. The original electrons, however, travel along the open field lines, and about 50 to $5000 \mathrm{~km}$ above the neutron star they produce a cone of emission by the means of a highly coherent process called the curvature radiation. If the magnetic axis is inclined to the rotational axis, this cone of emission sweeps around as the pulsar is rotating, and an observer will detect a pulse of radiation only once per pulsar rotation. This model is commonly known as the "lighthouse model".

The properties of the radio emission of pulsars are still an object of intense study. In general, when averaging a large number (more than a few thousand) of individual pulses (i.e., rotations) the pulsar's profile shape (the 


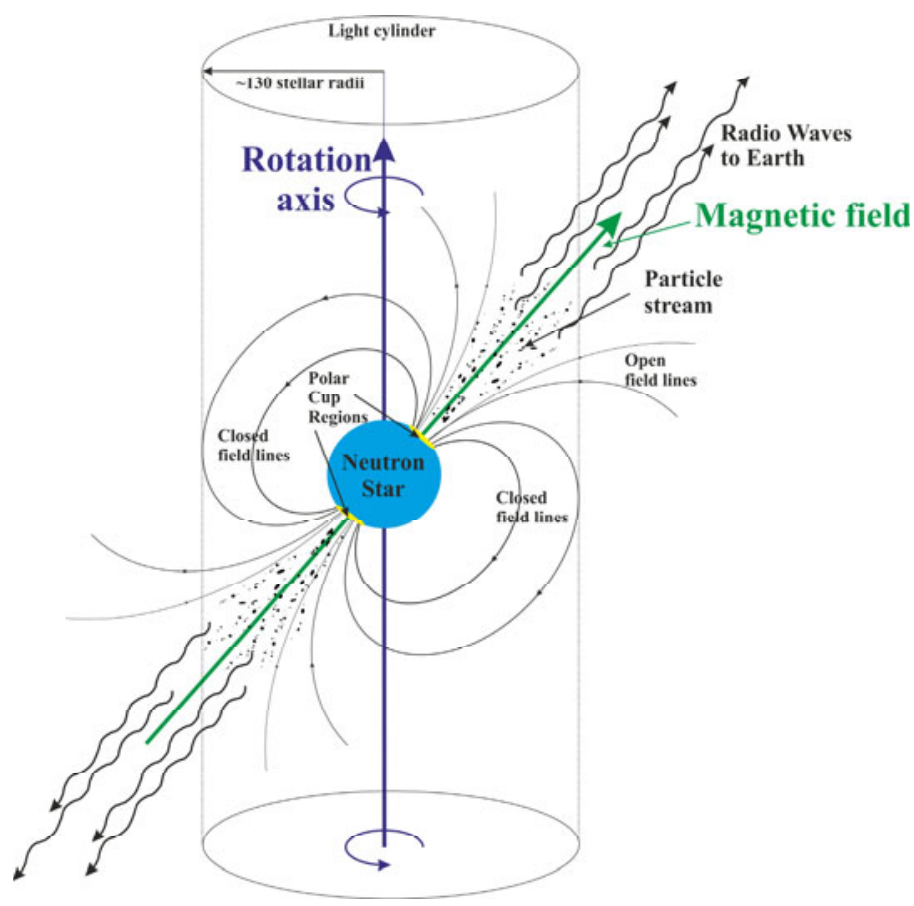

Fig. 5. The immediate environment of a neutron star.

intensity versus time light-curve for a single sweep of the pulsar's beam) remains stable, however the pulse shapes from individual rotations can look very different and they can exhibit a number of effects. One of the more successful models explaining various phenomena of pulsar radio emission is the "rotating carousel model" which was initially proposed by Ruderman and Sutherland (1975). This model explains the appearance of a phenomenon called the drifting subpulses (see, e.g., Gil and Sendyk 2003, and references therein). The pulsar emission is also frequency dependent, because for most of these objects the profiles observed at lower frequencies are broader than at high radio frequencies. This can be attributed to the so-called "radius-tofrequency mapping" (Kijak and Gil 2003) and can be explained by the fact that the radiation at lower frequencies originates higher in the magnetosphere, where the dipolar shape of the last open magnetic field line makes the cone of emission broader. At the same time, the higher frequency emissions require greater curvature of the magnetic field lines, hence they are emitted closer to the surface of the neutron star.

All of the above was inferred from the study of both average pulse shapes, as well as the shapes of individual pulses, yet until now there is no single model that would explain all the observed phenomena, such as the 
above-mentioned drifting subpulses, the micro- and nano-structure, the giant pulses, and nulling (see Lorimer and Kramer 2005, and references therein for a short but comprehensible description of these). The only way to find additional constraints on the currently accepted models is by further research, possibly at the observing frequencies that were not thoroughly explored before (like the LOFAR frequency range), and with sensitivity that was not possible until now.

\subsection{Pulsars as tools for the study of interstellar medium}

Pulsars are interesting objects of study by themselves, but as we mentioned at the beginning of this section they can also be used as tools for other fields of astrophysical research. One of these is definitely the study of the interstellar medium, thanks to some phenomena compiled in Fig. 6.

Radiation emitted by the pulsar propagates through the ISM which is composed of the neutral atoms (mainly hydrogen), but also contains a significant fraction of ionized material. For radio waves the most important is the interaction with the free electrons in the ISM. These cause few effects; the most important is probably the dispersion of the radio waves. In the dispersive medium the propagation velocity is frequency (wavelength) dependent. For pulsars this means that the radiation which is emitted at all frequencies in the same instant will not reach the observer at the same time, namely the pulse will appear at high frequency first, and at lower frequency only after some delay. The amount of the delay is strictly bound to the column density of the electrons along the line-of-sight (this column density is usually called the dispersion measure of the pulsar), and the measurement of this quantity can be used to estimate the density of the ISM, if we know the distance to the pulsar by other means. For more distant neutron stars, however, the situation is usually reversed - we do not know the distance, but using a model of galactic electron distribution (such as NE2001 model by Cordes and Lazio 2002) and measuring the value of the dispersion measure we can infer the
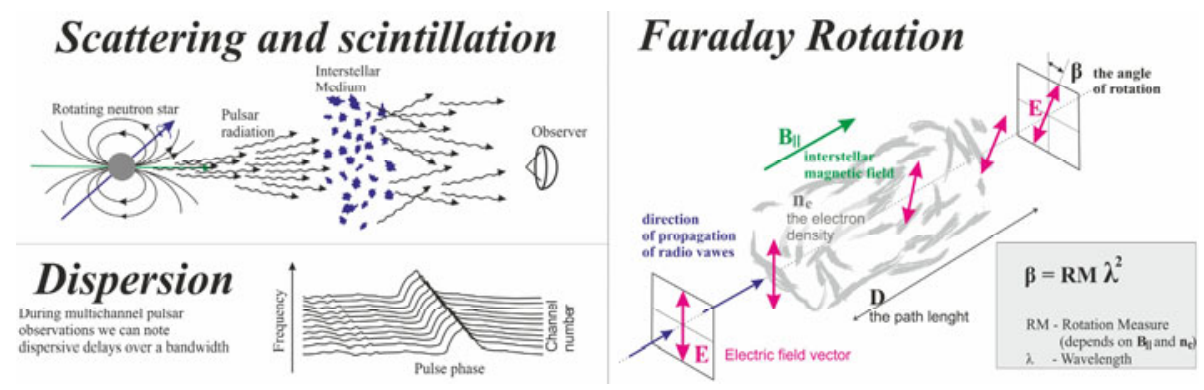

Fig. 6. Explanation of the phenomena associated with pulsar signal propagation through Interstellar Medium. 
pulsar distance. For most pulsars this is the only means to estimate their distances (and subsequently their positions in the Galaxy).

The next two effects observed in pulsars at radio-wave frequencies are the interstellar scattering and scintillation (see Rickett (1990) for a review of the theory). These effects originate due to the density fluctuations and turbulent nature of the ISM. The density fluctuations along the line-of-sight cause the pulsar's radiation to undergo scattering and as a result the radiation that reaches the observer does not travel along a single geometrical path. This causes the pulsar profiles to attain the so-called scattering tails, which are "smearing" the pulsar emission over a certain interval (see, e.g., the profile of PSR B2255+58 in Fig. 7) - these tails roughly resemble an exponential decay. Since the properties of the scattering in the ISM are frequency dependent, the observed amount of pulse broadening will also vary with frequency, roughly as $\sim v^{-4}$ (see Lewandowski et al. (2013) for a recent study of this dependence).

The interstellar scintillation is an effect which is similar in nature to the twinkling star phenomenon (known as atmospheric seeing in astrophysics). The pulsar radiation propagating through the ISM can be seen as a flat wavefront. When such a wavefront traverses through a region of electron density
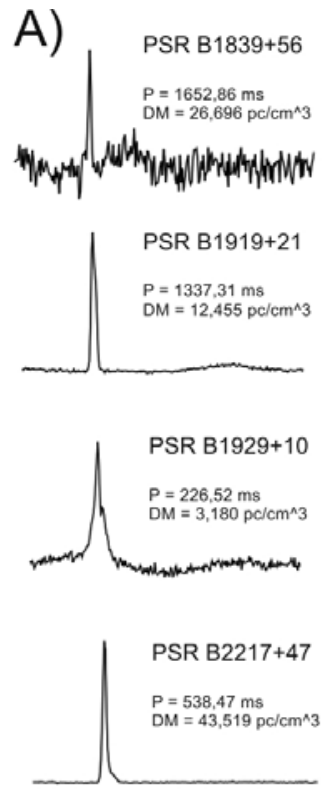
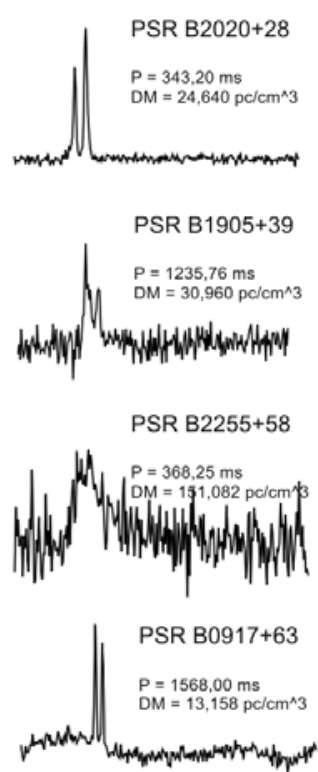

B)

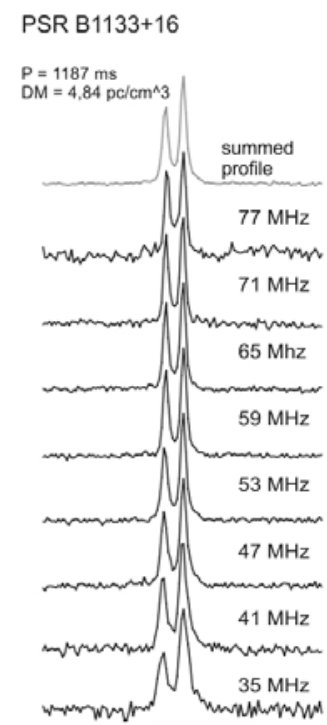

Fig. 7: (A) Examples of pulsar profiles obtained with LOFAR pilot surveys taken from Coenen et al. (2014); (B) Pulsar profiles obtained with LBA part of LOFAR taken from Stappers et al. (2011). Below the catalogue name of the pulsar its period (P) and dispersion measure (DM) are given. 
fluctuations it gets disturbed, which causes it to interfere with itself. At the observer's distance one can say that this interference (along with the diffraction and refraction effects) causes a specific pattern of amplification and dampening of the pulsar signal. As the Earth is moving through that pattern an observer will detect variations in the intensity of the received radiation. Since the amount of the wavefront disturbance depends on the frequency of the radiation, the diffraction patterns will vary depending on the observing frequency, and so will be the scintillation parameters - its timescales, decorrelation bandwidths, and modulation strength (see, e.g., Lewandowski et al. 2011).

Investigation of both scattering and scintillation properties of the incoming pulsar radiation, and especially the frequency scaling of their various parameters is virtually the only means to study the turbulent properties of the ISM. These in turn are important to our understanding of the structure of the galaxy, the galactic power sources, and the details of star formation.

Observations of pulsars can also broaden our knowledge about the galactic magnetic fields. Pulsar radio emission, due to its origin, is usually highly polarized. The polarized radiation then travels through the magnetic field of the Galaxy, undergoing the process known as Faraday rotation, which changes the position angle of the polarized pulsar signal. With the help of multi-frequency polarization measurements it is possible to estimate the strength of the field that affects the radiation (see, e.g., Han 2004).

All of the phenomena described above represent only a small fraction of our knowledge about pulsars and neutron stars in general, and in no way should be considered complete. We decided to focus on these selected topics, since the research in the mentioned fields of the pulsar astrophysics will be the main focus of our studies using the Polish LOFAR stations. For a more complete review of the current state of pulsar astronomy and its observational aspects see Lorimer and Kramer (2005), and the references therein.

\section{PROSPECTS FOR PULSAR INVESTIGATIONS WITH LOFAR}

Transient sources are one of the Key Projects of LOFAR. Under its remit come all time-variable astronomical radio sources, including pulsars (Grießmeier et al. 2012). In these kinds of radio sources, LOFAR gives us a chance to make observations at very short time scales. Time resolution depends on the sampling rate which for LOFAR observations is up to $200 \mathrm{MHz}$.

\subsection{Survey of galactic and extragalactic pulsars}

The spectrum of radio emissions from pulsars exhibits a steep shape with a flux density maximum in the $100-200 \mathrm{MHz}$ range (Lorimer and Kramer 2005). This fact together with the high sensitivity of LOFAR (see Sec- 
tion 3.2) makes it the best observational tool for pulsar investigations, including all-sky pulsar surveys. According to the luminosity function for pulsars (Lyne et al. 1998) and first results obtained with this instrument, LOFAR is able to measure the pulsar luminosity function definitely better than previous surveys. The sophisticated simulations made by van Leeuwen and Stappers (2006) and latter observations (see Stappers et al. 2011) showed that LOFAR is able to detect hundreds of new Galactic pulsars as well as any extragalactic pulsars.

\subsubsection{Examples of observations obtained with LOFAR}

The results of pilot surveys presented by Coenen et al. (2014) showed the high effectiveness of the multibeam method of observation used. Figure 7 shows examples of pulsar profiles obtained during the survey mentioned above.

The outcomes of broadband low-frequency observations of PSR B0943+10 (Bilous et al. 2014) resulted in a lot of new information about this peculiar pulsar. These results allow us to hope for a remarkable progress in the study of individual particularly interesting pulsars.

The most recent results of the pulsars observations carried out using the LOFAR telescope outlined by Noutsos et al. (2015) focus on the polarimetric properties of emissions of 20 bright pulsars. The authors presented an analysis of the highest quality polarization profiles ever obtained at frequencies below $200 \mathrm{MHz}$.

\subsection{The study of the physics of pulsar radio emissions}

As we mentioned in the previous section, the specifics of the pulsar radio emission mechanism still remain largely unknown, and there is no single theoretical model that would be able to explain all the observed phenomena. The only way to overcome this problem is by further studies of pulsar emission properties and that is best done by observations of individual pulses from pulsars.

While a single LOFAR station does not have enough sensitivity to detect individual pulses from a large number of objects, we still should be able to observe a few tens of sources in this manner (see Stappers et al. 2007). For these object we intend to analyze the data and especially to search for the evidence - and properties - of the drifting subpulses phenomenon, or any other manifestation of the rotating carousel model (Ruderman and Sutherland 1975). This is usually done by the means of Fourier-based analysis of series of individual pulses (see, e.g., Weltevrede et al. 2007, Honnappa et al. 2012) to detect periodic effects. This kind of research at low radio frequencies (i.e., in the LOFAR frequency range) was until now performed only occasionally. 
Another phenomenon that has not been thoroughly studied at low frequencies is the microstructure - a phenomenon showing that at least in some pulsars the observed pulses contain smaller structures with timescales in the range between a few to a few hundred microseconds (see, e.g., Kramer et al. 2002). It is believed that these microstructures are an imprint of the most basic pulsar emission phenomena, and a detailed study of these will definitely help us understand the underlying physics of pulsar radio emissions.

\subsection{Studies of interstellar medium (ISM)}

As we mentioned in Section 3, the main propagation effects observed in pulsar data and caused by ionized matter of the interstellar medium are the dispersion, scattering, scintillation, and Faraday rotation. Observations of these phenomena are definitely possible in the LOFAR frequency range, as was demonstrated by Stappers et al. (2011) or Bilous et al. (2014). In our research we want to focus mainly on the long-term monitoring projects (lasting from a few months to several years) of a few selected sources, which will help us to improve our understanding of the properties of the interstellar medium. These projects will include the monitoring of the dispersion measure variations, as well as the properties of the interstellar scattering: both the strength of scattering variations as well as its frequency scaling. This kind of research will help us to address the issues of anisotropy and inhomogeneity of the ISM (see Lewandowski et al. 2013, and references therein) which apparently influence the observed properties of scattering at timescales of weeks to months, and have been until now virtually not studied at all. Hopefully these studies will allow us to build upon the current models of scattering and scintillation, which usually assume an isotropic and homogenous interstellar medium, which has been proven to be far from the truth, at least for a number of pulsars.

The long term monitoring of scintillation parameters of pulsars is especially important, since - as we mentioned in Section 3 - the measured values of the scintillation parameters depend on the velocity of the observer. Since we are observing from the surface of Earth as it orbits the Sun, the actual speed at which the observer moves through the scintillation patter will vary (by a maximum of $\pm 30 \mathrm{~km} / \mathrm{s}$ ), which is a substantial amount. To disentangle the Earth's motion from the observed scintillation results, and ascertain the actual properties of the ISM, one needs at least one full year of observations (see, e.g., Lewandowski et al. 2011). Such an observing project would be almost impossible to perform with any of the currently operational large radio telescopes, due to the high observing time demand, while for a single LOFAR station this should not be a problem. 


\subsection{Ionospheric research}

The signals emitted by pulsars can be distorted in various ways by the Earth's ionosphere (see Fig. 1). This gives us a unique opportunity to study the structure of the ionosphere in a completely new and independent way. A few possibilities including Faraday depth measurement techniques as described in detail by Sotomayor-Beltran et al. (2013).

\section{CONCLUSIONS}

The LOFAR is working and first results for pulsars survey were already published (Coenen et al. 2014, Bilous et al. 2014, Noutsos et al. 2015). It shows the great potential of the telescope at single station mode observations. The building of three new stations in Poland is in progress and a few more in the rest of Europe are also in the plans. The revolutionary design of this instrument is an important step towards the future of radio astronomy and space research. Inherent is also the possibility of making significant progress in a wide range of technical areas.

The specialized equipment hardware and software (ARTEMIS) which is continuously being further developed and improved raises hopes for great possibilities in the future. For example: automated observations and instrument-internal analysis will significantly increase the efficiency of measurements. More precise coherent de-dispersion methods will give us the chance to search for signal disturbances in the Earth's ionosphere.

The LOFAR single station mode provides the opportunity to put particular attention on long-term monitoring of interesting pulsars, as well as single pulse and variability studies over very wide bandwidths investigations.

POLFAR - the Polish part of LOFAR (Krankowski et al. 2014) is not only an opportunity to conduct independent research but most of all it will lead to a significant expansion of the possibilities of the entire LOFAR interferometer. Beginning from autumn 2015, POLFAR will be the biggest radio astronomical tool dedicated and available for astronomers and space science researchers in Poland.

Acknowledgments. The Polish LOFAR stations have been funded by the Polish Ministry of Science and Higher Education; the funds of the large research infrastructure "Construction of the station Polish European LOFAR radio interferometer" (grant No. 6339/IA/158/2013.1). This paper was partially supported by the grants DEC-2012/05/B/ST9/03924 and DEC2013/09/B/ST9/02177 of the Polish National Science Centre.

LB also would like to thank Professor Dieter Bilitza for the language correction. 


\section{References}

Anderson, J.M. (2012), The LuMP data recording system for single-station LOFAR use. In: German Long Wavelength Consortium (GLOW) Annual Meeting, 18-19 June 2012; Bielefeld, Germany, http://www2.physik.unibielefeld.de/ fileadmin/user_upload/workshops/GlowAssembly2012/anderson_LuMP.pdf.

Bilous, A.V., J.W.T. Hessels, V.I. Kondratiev, J. van Leeuwen, B.W. Stappers, P. Weltevrede, H. Falcke, T. E. Hassall, M. Pilia, E. Keane, M. Kramer, J.-M. Grießmeier, and M. Serylak (2014), LOFAR observations of PSR B0943+10: profile evolution and discovery of a systematically changing profile delay in bright mode, Astron. Astrophys. 572, A52, DOI: 10.1051/ 0004-6361/201424425.

Broekema, C. (2013), COBALT - A new correlator for LOFAR, ASTRON The Netherlands Institute for Radio Astronomy.

Coenen, T., J. van Leeuwen, J.W.T. Hessels, B.W. Stappers, V.I. Kondratiev, A. Alexov, R.P. Breton, A. Bilous, S. Cooper, H. Falcke, R.A. Fallows, V. Gajjar, J.-M. Grießmeier, T.E. Hassall, A. Karastergiou, E.F. Keane, M. Kramer, M. Kuniyoshi, A. Noutsos, S. Osłowski, M. Pilia, M. Serylak, C. Schrijvers, C. Sobey, S. ter Veen, J. Verbiest, P. Weltevrede, S. Wijnholds, K. Zagkouris, A.S. van Amesfoort, J. Anderson, A. Asgekar, I.M. Avruch, M.E. Bell, M.J. Bentum, G. Bernardi, P. Best, A. Bonafede, F. Breitling, J. Broderick, M. Brüggen, H.R. Butcher, B. Ciardi, A. Corstanje, A. Deller, S. Duscha, J. Eislöffel, R. Fender, C. Ferrari, W. Frieswijk, M.A. Garrett, F. de Gasperin, E. de Geus, A.W. Gunst, J.P. Hamaker, G. Heald, M. Hoeft, A. van der Horst, E. Juette, G. Kuper, C. Law, G. Mann, R. McFadden, D. McKay-Bukowski, J.P. McKean, H. Munk, E. Orru, H. Paas, M. Pandey-Pommier, A.G. Polatidis, W. Reich, A. Renting, H. Röttgering, A. Rowlinson, A.M.M. Scaife, D. Schwarz, J. Sluman, O. Smirnov, J. Swinbank, M. Tagger, Y. Tang, C. Tasse, S. Thoudam, C. Toribio, R. Vermeulen, C. Vocks, R.J. van Weeren, O. Wucknitz, P. Zarka, and A. Zensus (2014), The LOFAR pilot surveys for pulsars and fast radio transients, Astron. Astrophys. 570, A60, DOI: 10.1051/0004$6361 / 201424495$.

Cordes, J.M., and T.J.W. Lazio (2002), NE2001.I. A new model for the galactic distribution of free electrons and its fluctuations, arXiv:astro-ph/0207156.

de Vos, M., A.W. Gunst, and R. Nijboer (2009), The LOFAR telescope: System architecture and signal processing, Proc. IEEE 97, 8, 1431-1437, DOI: 10.1109/JPROC.2009.2020509.

Ewen, H.I., and E.M. Purcell (1951), Observation of a line in the galactic radio spectrum: Radiation from galactic hydrogen at 1,420 Mc./sec, Nature 168, 4270, 356-356, DOI: 10.1038/168356a0.

Gil, J.A., and M. Sendyk (2003), Drifting subpulses in PSR B0943+10, Astrophys. J. 585, 1, 453-463, DOI: 10.1086/346079. 
Gil, J., G.I. Melikidze, and U. Geppert (2003), Drifting subpulses and inner acceleration regions in radio pulsars, Astron. Astrophys. 407, 1, 315-324, DOI: 10.1051/0004-6361:20030854.

Goldreich, P., and W.H. Julian (1969), Pulsar electrodynamics, Astrophys. J. 157, 2P1, 869-880, DOI: 10.1086/150119.

Grießmeier, J.-M., P. Zarka, and M. Tagger (2012), Radioastronomy with LOFAR, C. R. Phys. 13, 1, 23-27, DOI: 10.1016/j.crhy.2011.11.002.

Han, J.L. (2004), The large-scale magnetic field structure of our galaxy: Efficiently deduced from pulsar rotation measures. In: B. Uyaniker, W. Reich, and R. Wielebinski (eds.), The Magnetized Interstellar Medium, Copernicus GmbH, Katlenburg-Lindau, 3-12.

Hessels, J.W.T., B. Stappers, A. Alexov, T. Coenen, T. Hassall, A. Karastergiou, V. Kondratiev, M. Kramer, J. van Leeuwen, J.D. Mol, A. Noutsos, and P. Weltevrede (2010), Early pulsar observations with LOFAR. In: Proc. ISKAF2010 Science Meeting, arXiv:1009.1758.

Hewish, A., S.J. Bell, J.D.H. Pilkington, P.F. Scott, and R.A. Collins (1968), Observation of a rapidly pulsating radio source, Nature $217,5130,709-713$, DOI: $10.1038 / 217709 \mathrm{a} 0$.

Honnappa, S., W. Lewandowski, J. Kijak, A.A. Deshpande, J. Gil, O. Maron, and A. Jessner (2012), Single-pulse analysis of PSR B1133+16 at $8.35 \mathrm{GHz}$ and carousel circulation time, Mon. Not. Roy. Astron. Soc. 421, 3, 19962001, DOI: 10.1111/j.1365-2966.2012.20424.x.

Hulse, R.A., and J.H. Taylor (1975), Discovery of a pulsar in a binary system, The Astrophys. J. 195, 2, L51-L53, DOI: 10.1086/181708.

Jansky, K.G. (1933), Radio waves from outside the solar system, Nature 132, 3323, 66-66, DOI: $10.1038 / 132066 \mathrm{a} 0$.

Kijak, J., and J. Gil (2003), Radio emission altitude in pulsars, Astron. Astrophys. 397, 3, 969-972, DOI: 10.1051/0004-6361:20021583.

Kramer, M., S. Johnston, and W. van Straten (2002), High-resolution single-pulse studies of the Vela pulsar, Mon. Not. Roy. Astron. Soc. 334, 3, 523-532, DOI: 10.1046/j.1365-8711.2002.05478.x.

Krankowski, A., L. Błaszkiewicz, K. Otmianowska-Mazur, M. Soida, H. Rothkaehl, and B. Atamaniuk (2014), POLFAR - Polish incarnation of the LOFAR. Scientific objectives and system realization. In: Proc. 20th Int. Conf. on Microwaves, Radar and Wireless Communication (MIKON), 16-18 June 2014, Gdańsk, Poland, DOI: 10.1109/MIKON.2014.6899926.

Lewandowski, W., J. Kijak, Y. Gupta, and K. Krzeszowski (2011), Diffractive and refractive timescales at $4.8 \mathrm{GHz}$ in PSR B0329+54, Astron. Astrophys. 534, A66, DOI: 10.1051/0004-6361/201116850.

Lewandowski, W., M. Dembska, J. Kijak, and M. Kowalińska (2013), Pulse broadening analysis for several new pulsars and anomalous scattering, Mon. Not. Roy. Astron. Soc. 434, 1, 69-83, DOI: 10.1093/mnras/stt989. 
Lorimer, D.R., and M. Kramer (2005), Handbook of Pulsar Astronomy, Cambridge University Press, Cambridge.

Lyne, A.G., R.N. Manchester, D.R. Lorimer, M. Bailes, N. D’Amico, T.M. Tauris, S. Johnston, J.F. Bell, and L. Nicastro (1998), The Parkes southern pulsar survey - II. Final results and population analysis, Mon. Not. Roy. Astron. Soc. 295, 4, 743-755, DOI: 10.1046/j.1365-8711.1998.01144.x.

Melikidze, G.I., D. Mitra, and J. Gil (2014), On the adiabatic walking of plasma waves in a pulsar magnetosphere, Astrophys. J. 794, 2, 105, DOI: 10.1088/ 0004-637X/794/2/105.

Noutsos, A., C. Sobey, V.I. Kondratiev, P. Weltevrede, J.P.W. Verbiest, A. Karastergiou, M. Kramer, M. Kuniyoshi, A. Alexov, R.P. Breton, A.V. Bilous, S. Cooper, H. Falcke, J.-M. Grießmeier, T.E. Hassall, J.W.T. Hessels, E.F. Keane, S. Osłowski, M. Pilia, M. Serylak, B.W. Stappers, S. ter Veen, J. van Leeuwen, K. Zagkouris, K. Anderson, L. Bähren, M. Bell, J. Broderick, D. Carbone, Y. Cendes, T. Coenen, S. Corbel, J. Eislöffel, R. Fender, H. Garsden, P. Jonker, C. Law, S. Markoff, J. Masters, J. Miller-Jones, G. Molenaar, R. Osten, M. Pietka, E. Rol, A. Rowlinson, B. Scheers, H. Spreeuw, T. Staley, A. Stewart, J. Swinbank, R. Wijers, R. Wijnands, M. Wise, P. Zarka, and A. van der Horst (2015), Pulsar polarisation below $200 \mathrm{MHz}$ : Average profiles and propagation effects, Astron. Astrophys. 576, A62, DOI: 10.1051/0004-6361/201425186.

Pines, D., and M.A. Alpar (1985), Superfluidity in neutron stars, Nature 316, 6023, 27-32, DOI: $10.1038 / 316027 \mathrm{a} 0$.

Rickett, B.J. (1990), Radio propagation through the turbulent interstellar plasma, Ann. Rev. Astron. Astrophys. 28, 561-605, DOI: 10.1146/annurev.aa.28. 090190.003021.

Ruderman, M.A., and P.G. Sutherland (1975), Theory of pulsars: Polar gaps, sparks, and coherent microwave radiation, Astrophys. J. 196, 51-72, DOI: 10.1086/ 153393.

Serylak, M., A. Karastergiou, C. Williams, W. Armour, M. Giles, and LOFAR Pulsar Working Group (2012), Observations of transients and pulsars with LOFAR international stations and the ARTEMIS backend, Proc. Int. Astron. Un. 8, S291, 492-494, DOI: 10.1017/S1743921312024623.

Shapiro, S.L., and S.A. Teukolsky (1983), Black Holes, White Dwarfs, and Neutron Stars. The Physics of Compact Objects, Wiley Interscience, New York.

Sotomayor-Beltran, C., C. Sobey, J.W.T. Hessels, G. de Bruyn, A. Noutsos, A. Alexov, J. Anderson, A. Asgekar, I.M. Avruch, R. Beck, M.E. Bell, M.R. Bell, M.J. Bentum, G. Bernardi, P. Best, L. Birzan, A. Bonafede, F. Breitling, J. Broderick, W.N. Brouw, M. Brüggen, B. Ciardi, F. de Gasperin, R.-J. Dettmar, A. van Duin, S. Duscha, J. Eislöffel, H. Falcke, R.A. Fallows, R. Fender, C. Ferrari, W. Frieswijk,M.A. Garrett, J. Grießmeier, T. Grit, A.W. Gunst, T.E. Hassall, G. Heald, M. Hoeft, A. Horneffer, M. Iacobelli, E. Juette, A. Karastergiou, E. Keane, J. Kohler, M. Kramer, 
V.I. Kondratiev, L.V.E. Koopmans, M. Kuniyoshi, G. Kuper, J. van Leeuwen, P. Maat, G. Macario, S. Markoff, J. P. McKean, D.D. Mulcahy, H. Munk, E. Orru, H. Paas, M. Pandey-Pommier, M. Pilia, R. Pizzo, A.G. Polatidis, W. Reich, H. Röttgering, M. Serylak, J. Sluman, B.W. Stappers, M. Tagger, Y. Tang, C. Tasse, S. ter Veen, R. Vermeule ${ }^{3}$, R.J. van Weeren, R.A.M.J. Wijers, S.J. Wijnholds, M.W. Wise, O. Wucknitz, S. Yatawatta, and P. Zarka (2013), Calibrating high-precision Faraday rotation measurements for LOFAR and the next generation of low-frequency radio telescopes, Astron. Astrophys. 552, A58, DOI: 10.1051/0004-6361/ 201220728.

Stappers, B.W., A.G.J. van Leeuwen, M. Kramer, D. Stinebring, and J. Hessels (2007), LOFAR: A powerful tool for pulsar studies. In: W. Becker and H.H. Huang. (eds.), Proc. 363rd WE-Heraeus Seminar on Neutron Stars and Pulsars: 40 Years After the Discovery, 14-19 May 2006, Bad Honnef, Germany, Max Planck Institut für Extraterrestrische Physik, MPE Report 291, 100-103.

Stappers, B.W., J.W.T. Hessels, A. Alexov, K. Anderson, T. Coenen, T. Hassall, A. Karastergiou, V.I. Kondratiev, M. Kramer, J. van Leeuwen, J.D. Mol, A. Noutsos, J.W. Romein, P. Weltevrede, R. Fender, R.A.M. J. Wijers, L. Bähren, M.E. Bell, J. Broderick, E.J. Daw, V.S. Dhillon, J. Eislöffel, H. Falcke, J. Griessmeier, C. Law, S. Markoff, J.C.A. Miller-Jones, B. Scheers, H. Spreeuw, J. Swinbank, S. ter Veen, M.W. Wise, O. Wucknitz, P. Zarka, J. Anderson, A. Asgekar, I.M. Avruch, R. Beck, P. Ben nema, M.J. Bentum, P. Best, J. Bregman, M. Brentjens, R.H. van de Brink, P.C. Broekema, W.N. Brouw, M. Brüggen, A.G. de Bruyn, H.R. Butcher, B. Ciardi, J. Conway, R.-J. Dettmar, A. van Duin, J. van Enst, M. Garrett, M. Gerbers, T. Grit, A. Gunst, M.P. van Haarlem, J.P. Hamaker, G. Heald, M. Hoeft, H. Holties, A. Horneffer, L.V.E. Koopmans, G. Kuper, M. Loose, P. Maat, D. McKay-Bukowski, J.P. McKean, G. Miley, R. Morganti, R. Nijboer, J.E. Noordam, M. Norden, H. Olofsson, M. PandeyPommier, A. Polatidis, W. Reich, H. Röttgering, A. Schoenmakers, J. Sluman, O. Smirnov, M. Steinmetz, C.G. M. Sterks, M. Tagger, Y. Tang, R. Vermeulen, N. Vermaas, C. Vogt, M. de Vos, S.J. Wijnholds, S. Yatawatta, and A. Zensus (2011), Observing pulsars and fast transients with LOFAR, Astron. Astrophys. 530, A80, DOI: 10.1051/0004-6361/ 201116681.

Szary, A., G.I. Melikidze, and J. Gil (2015), Two modes of partially screened gap, Mon. Not. Roy. Astron. Soc. 447, 3, 2295-2306, DOI: 10.1093/mnras/ stu2622.

van der Tol, S., and A.-J. van der Veen (2007), Ionospheric calibration for the LOFAR radiotelescope. In: Proc. Int. Symp. on Signals, Circuits and Systems, ISSCS 2007, 13-14 July 2007, Iasi, Romania (Vol. 2), DOI: 10.1109/ ISSCS.2007.4292761. 
van Haarlem, M.P., M.W. Wise, A.W. Gunst, G. Heald, J.P. McKean, J.W.T. Hessels, A.G. de Bruyn, R. Nijboer, J. Swinbank, R. Fallows, M. Brentjens, A. Nelles, R. Beck, H. Falcke, R. Fender, J. Hörandel, L.V.E. Koopmans, G. Mann, G. Miley, H. Röttgering, B.W. Stappers, R.A.M.J. Wijers, S. Zaroubi, M. van den Akker, A. Alexov, J. Anderson, K. Anderson, A. van Ardenne, M. Arts, A. Asgekar, I.M. Avruch, F. Batejat, L. Bähren, M.E. Bell, M.R. Bell, I. van Bemmel, P. Bennema, M.J. Bentum, G. Bernardi, P. Best, L. Bîrzan, A. Bonafede, A.-J. Boonstra, R. Braun, J. Bregman, F. Breitling, R.H. van de Brink, J. Broderick, P.C. Broekema, W.N. Brouw, M. Brüggen, H.R. Butcher, W. van Cappellen, B. Ciardi, T. Coenen, J. Conway, A. Coolen, A. Corstanje, S. Damstra, O. Davies, A.T. Deller, R.-J. Dettmar, G. van Diepen, K. Dijkstra, P. Donker, A. Doorduin, J. Dromer, M. Drost, A. van Duin, J. Eislöffel, J. van Enst, C. Ferrari, W. Frieswijk, H. Gankema, M.A. Garrett, F. de Gasperin, M. Gerbers, E. de Geus, J.-M. Grießmeier, T. Grit, P. Gruppen, J.P. Hamaker, T. Hassall, M. Hoeft, H.A. Holties, A. Horneffer, A. van der Horst, A. van Houwelingen, A. Huijgen, M. Iacobelli, H. Intema, N. Jackson, V. Jelic, A. de Jong, E. Juette, D. Kant, A. Karastergiou, A. Koers, H. Kollen, V.I. Kondratiev, E. Kooistra, Y. Koopman, A. Koster, M. Kuniyoshi, M. Kramer, G. Kuper, P. Lambropoulos, C. Law, J. van Leeuwen, J. Lemaitre, M. Loose, P. Maat, G. Macario, S. Markoff, J. Masters, R.A. McFadden, D. McKay-Bukowski, H. Meijering, H. Meulman, M. Mevius, E. Middelberg, R. Millenaar, J.C.A. Miller-Jones, R.N. Mohan, J.D. Mol, J. Morawietz, R. Morganti, D.D. Mulcahy, E. Mulder, H. Munk, L. Nieuwenhuis, R. van Nieuwpoort, J.E. Noordam, M. Norden, A. Noutsos, A.R. Offringa, H. Olofsson, A. Omar, E. Orrú, R. Overeem, H. Paas, M. Pandey-Pommier, V.N. Pandey, R. Pizzo, A. Polatidis, D. Rafferty, S. Rawlings, W. Reich, J.-P. de Reijer, J. Reitsma, G.A. Renting, P. Riemers, E. Rol, J.W. Romein, J. Roosjen, M. Ruiter, A. Scaife, K. van der Schaaf, B. Scheers, P. Schellart, A. Schoenmakers, G. Schoonderbeek, M. Serylak, A. Shulevski, J. Sluman, O. Smirnov, C. Sobey, H. Spreeuw, M. Steinmetz, C.G.M. Sterks, H.-J. Stiepel, K. Stuurwold, M. Tagger, Y. Tang, C. Tasse, I. Thomas, S. Thoudam, M.C. Toribio, B. van der Tol, O. Usov, M. van Veelen, A.-J. van der Veen, S. ter Veen, J.P.W. Verbiest, R. Vermeulen, N. Vermaas, C. Vocks, C. Vogt, M. de Vos, E. van der Wal, R. van Weeren, H. Weggemans, P. Weltevrede, S. White, S.J. Wijnholds, T. Wilhelmsson, O. Wucknitz, S. Yatawatta, P. Zarka, A. Zensus, and J. van Zwieten (2013), LOFAR: The LOw-Frequency ARray, Astron. Astrophys. 556, A2, DOI: 10.1051/0004-6361/201220873.

van Leeuwen, J., and B. Stappers (2006), Pulsar research with LOFAR, the first next-generation radio telescope. In: 26th Meeting of the IAU "On the Present and Future of Pulsar Astronomy”, 16-17 August 2006, Prague, Czech Republic.

van Straten, W., P. Demorest, and S. Osłowski (2012), Pulsar data analysis with PSRCHIVE, Astron. Res. Technol. 9, 3, 237-256, arXiv:1205.6276. 
Weber, F., R. Negreiros, and P. Rosenfield (2009), Neutron star interiors and the equation of state of superdense matter. In: W. Becker (ed.), Neutron Stars and Pulsars, Astrophysics and Space Science Library, Vol. 357, Springer, Berlin Heidelberg, 213-245, DOI: 10.1007/978-3-540-76965-1_0.

Weltevrede, P., B.W. Stappers, and R.T. Edwards (2007), The subpulse modulation properties of pulsars at $92 \mathrm{~cm}$ and the frequency dependence of subpulse modulation, Astron. Astrophys. 469, 2, 607-631, DOI: 10.1051/00046361:20066855.

Received 3 March 2015

Received in revised form 10 July 2015

Accepted 2 September 2015 